EXTENDED REPORT

\title{
Correlation of long term phenotypic and clinical outcomes following limbal epithelial transplantation cultivated on amniotic membrane in rabbits
}

\author{
S-E Ti, M Grueterich, E M Espana, A Touhami, D F Anderson, S C G Tseng
}

Br J Ophthalmol 2004;88:422-427. doi: 10.1136/bjo.2003.026054

See end of article for authors' affiliations

Correspondence to: Scheffer C G Tseng, MD, $\mathrm{PhD}$, Ocular Surface Center, 7000 SW 97th Avenue, Suite 213, Miami, FL 33173, USA;

stseng@ocularsurface.com

Accepted for publication 1 August 2003

\begin{abstract}
Aim: To determine the epithelial phenotype in rabbits with total limbal stem cell deficiency (LSCD) after reconstruction with autologous limbal epithelial stem cells ex vivo expanded on rabbit amniotic membrane (AM).

Methods: Left eyes of 52 rabbits were rendered total LSCD, verified by impression cytology. The fibrovascular pannus of each cornea was removed. Group I $(n=10)$ received rabbit AM transplantation alone, while groups II-IV ( $n=42)$ underwent transplantation of LSC cultured on rabbit AM (LSC-AM) from a small limbal biopsy taken from the right eye. Clinical outcome was graded as "success," "partial success," or "failure" depending on the corneal smoothness and avascularity. Epithelial phenotype was determined by immunostaining and graded as "corneal $(K)$," "conjunctival $(J)$," or "mixed (M)" depending on expression of K3 and Muc5AC.

Results: After 1 year follow up, group I showed 100\% failure and groups II-IV showed $26 \%$ success $(p<0.001)$. Clinical failure correlated with J phenotype $p=0.001)$, while clinical success correlated with $\mathrm{K}$ phenotype $p=0.01)$. When the phenotypic outcome was used for comparison, J phenotype was significantly high in group I ( $p=0.003)$, while K phenotype was significantly high in groups II-IV $(p<0.05)$. Conclusion: There is a strong correlation between clinical success and resultant corneal epithelial phenotype. Ex vivo expanded LSC can successfully reconstruct corneal surfaces with unilateral total LSCD.
\end{abstract}

$\mathrm{T}$ he integrity of the corneal epithelium depends on its epithelial stem cells located at the limbus. Limbal epithelial stem cells (LSC) serve as the ultimate source for maintenance and regeneration of the corneal epithelium. ${ }^{1-3}$ Damage or dysfunction of the limbus results in a state termed limbal stem cell deficiency (LSCD), which can be found by impression cytology in a number of ocular surface diseases. ${ }^{4}$ Restoration of corneal surfaces in patients with LSCD resorts to transplantation of autologous or allogeneic LSC. ${ }^{5}$

To avoid potential complications to the living donor eye in traditional autologous and allogeneic limbal transplantation, a new surgical procedure has successfully been practised in human patients with partial or total LSCD by expanding LSC ex vivo in culture from a small limbal biopsy. ${ }^{7-11}$ Undoubtedly, these human clinical experiences lend significant insight to our understanding of this cultivation technique.

To ascertain safety and clinical efficacy of this new surgical procedure, we have used an animal model to study culturing and surgical variables affecting the long term outcome following transplantation of ex vivo expanded LSC. ${ }^{12}$ We have identified that a single most important factor promoting clinical success is to apply an additional amniotic membrane (AM) as a temporary patch over the composite graft consisting of cultivated LSC on AM so that epithelialisation can be ensured at the early stage. ${ }^{12}$ After 1 year follow up, we verified that clinical outcome of success is correlated with the recovery of a normal corneal epithelial phenotype. The significance of these findings is further discussed.

\section{MATERIALS AND METHODS}

Materials

Monoclonal antibodies against keratin K3 (AE-5 1:100) were from ICN (Aurora, OH, USA) and goblet cell mucin (MUC
5AC 1:100) was a kind gift from Jacques Bara, MD, Paris, France. Other materials were obtained as reported. ${ }^{12}$

\section{Animals}

A total of 65 New Zealand White rabbits (divided into four study groups were included in this study protocol approved by the animal research committee of the University of Miami (No 01-127) and followed guidelines described in the ARVO statement for the use of animals in ophthalmic and vision research. After excluding 13 rabbits because of surgical complications, the remaining 52 rabbits were subdivided into four groups.

\section{Creation of total LSCD model}

The model of total LSCD was created in the left eye of all rabbits using a method previously described..$^{13}{ }^{14}$ The ocular surface became progressively conjunctivalised, with ingrowing fibrovascular pannus. Impression cytology was performed to confirm total LSCD using a previous method ${ }^{15}$ and based on a published diagnostic criterion.

\section{Preparation of rabbit AM}

Rabbit AM was harvested from 28 day pregnant does under sterile conditions, prepared and preserved as previously described. ${ }^{16}$

Abbreviations: $A M$, amniotic membrane; $A M T$, amniotic membrane transplantation; LSC, limbal epithelial stem cells; LSCD, limbal stem cell deficiency 

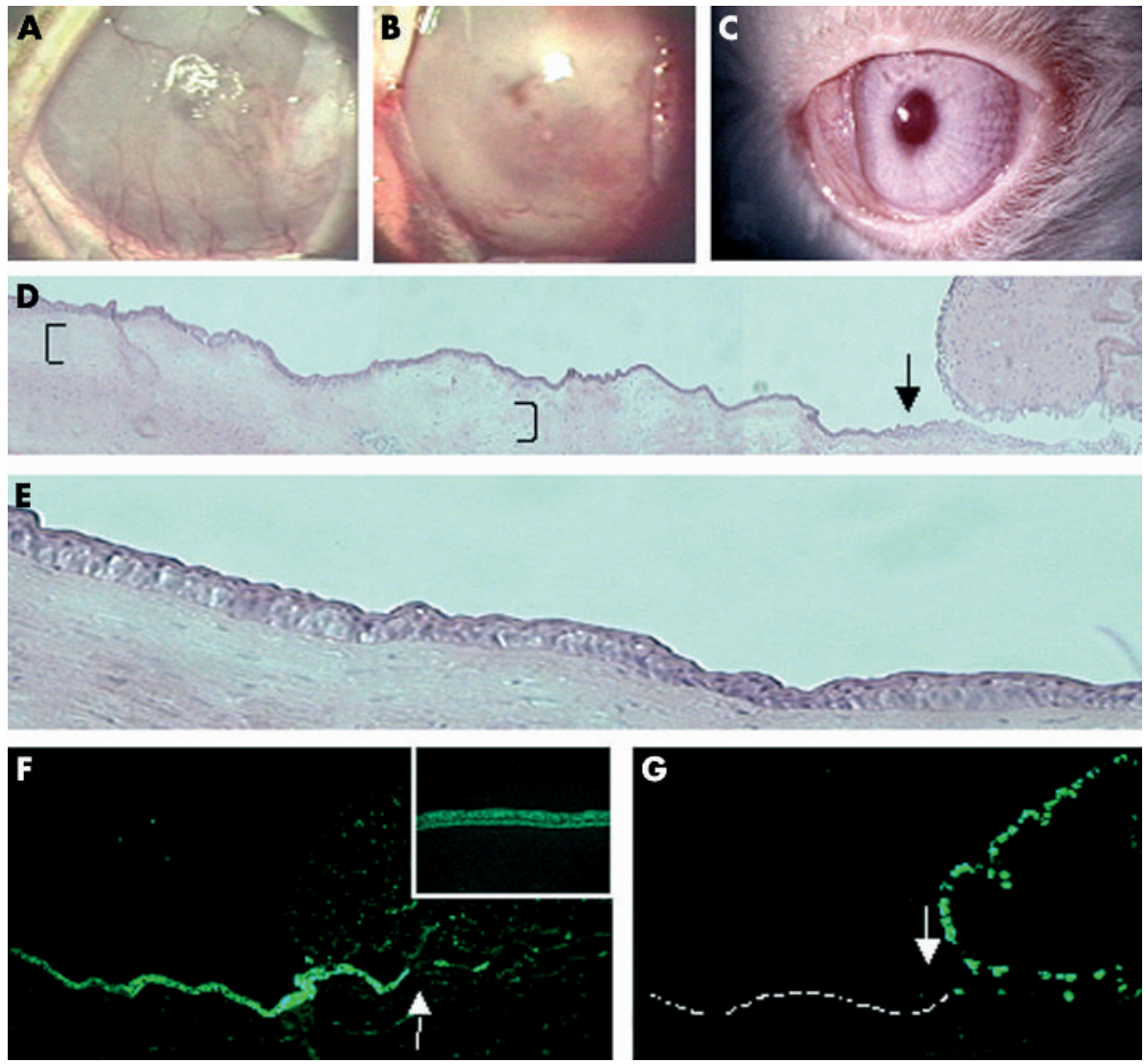

Figure 1 Correlation of clinical success with a corneal phenotype. preoperative appearance (A). After transplantation of ex vivo expanded LSC (B). Postoperative appearance (C). Haematoxylin and eosin staining (D and E). Immunostaining to K3 keratin (F) the entire corneal epithelium in inset, arrow from the limbal region). Immunostaining to MUC5AC in the conjunctival epithelium $(G$, arrow marks the limbus).

\section{Preparation of AM inserts, limbal biopsy, and culture on AM}

After thawing and rinsing in HBSS, the AM was fastened onto inserts. ${ }^{17} \mathrm{~A}$ limbal biopsy was performed in the normal right eye of each rabbit in groups II and III. The method of ex vivo expansion from the limbal biopsy specimen has been described. ${ }^{12}$

\section{Surgical transplantation}

The left eye of each rabbit in all groups underwent $360^{\circ}$ conjunctival peritomy followed by removal of the fibrovascular pannus. ${ }^{18}$ Group I $(\mathrm{n}=10)$ underwent rabbit AM transplantation (AMT) alone as previously described. ${ }^{19}$ Groups II-IV $(\mathrm{n}=42)$ underwent AMT with ex vivo expanded limbal epithelial cells and differences among them were previously reported. ${ }^{12}$

\section{Definition of clinical success}

Whenever the cornea became fully vascularised-that is, reverting to a preoperative appearance, the animal was killed and rated as a failure. Otherwise, all animals were followed up for more than 1 year before sacrifice. If the cornea regained its smoothness, avascularity, and clarity the animal was graded as a success. If the cornea showed a mixture of the above two features - that is, two quadrants or more of clear cornea, the eye was graded as a partial success.

\section{Phenotypic analysis}

Outcome was also measured by the epithelial phenotype of the transplanted corneas obtained after sacrifice by haematoxylin and eosin and periodic acid Schiff (PAS) staining and by immunofluorescence staining with the monoclonal antibody AE5 to $\mathrm{K} 3$ keratin $^{1}$ and with the monoclonal antibody AM3 to MUC5AC goblet cell mucin. ${ }^{18-20}$ Complete success was defined if the corneal epithelial phenotype was restored, as evidenced by the restoration of a stratified epithelium without goblet cell, which was positive to AE5 but negative to AM3 staining. Failure was defined if the resultant epithelium was of a conjunctival origin, as shown by the presence of goblet cells, which was negative to AE5 but positive to AM3 staining. In some eyes, a mixed phenotype was noted with the resultant epithelium showing a mixture of the above two phenotypes.

\section{Statistical analysis}

The comparison of the frequency of clinical outcomes of success, partial success, and failure or age and that of phenotypic outcomes of K, J, or M was performed by Fisher's exact test. The correlation of clinical outcomes with phenotypic outcomes was performed by two tailed $\chi^{2}$ test using Graph Pad InStat Version 3.05 (Graph Pad Software, Inc, CA, USA). A p value of $<0.05$ was considered as statistically significant.

\section{RESULTS}

\section{Clinical outcome}

The full clinical results have previously been reported. ${ }^{12}$ The cumulative percentage survival at 30 and 180 days was $10 \%$ and $0 \%$ for group 1, $64 \%$ and $21 \%$ for group II, $77 \%$ and $18 \%$ for group III, and $100 \%$ and $74 \%$ for group IV, respectively. The log rank test showed that group IV had statistically longer times to transplant failure (that is, significantly higher transplant success) than the other three surgical groups (all $\mathrm{p}<0.002$ ). The success rate was $0 \%$ in group I but $26 \%$ in groups II-IV $(\mathrm{p}<0.0001)$.

\section{Phenotypic outcome}

For groups II, III, and IV, corneas with clinical success were restored with a smooth and avascular surface and clear stroma (fig $1 \mathrm{~A}-\mathrm{C}$ ). Haematoxylin and eosin staining demon- 

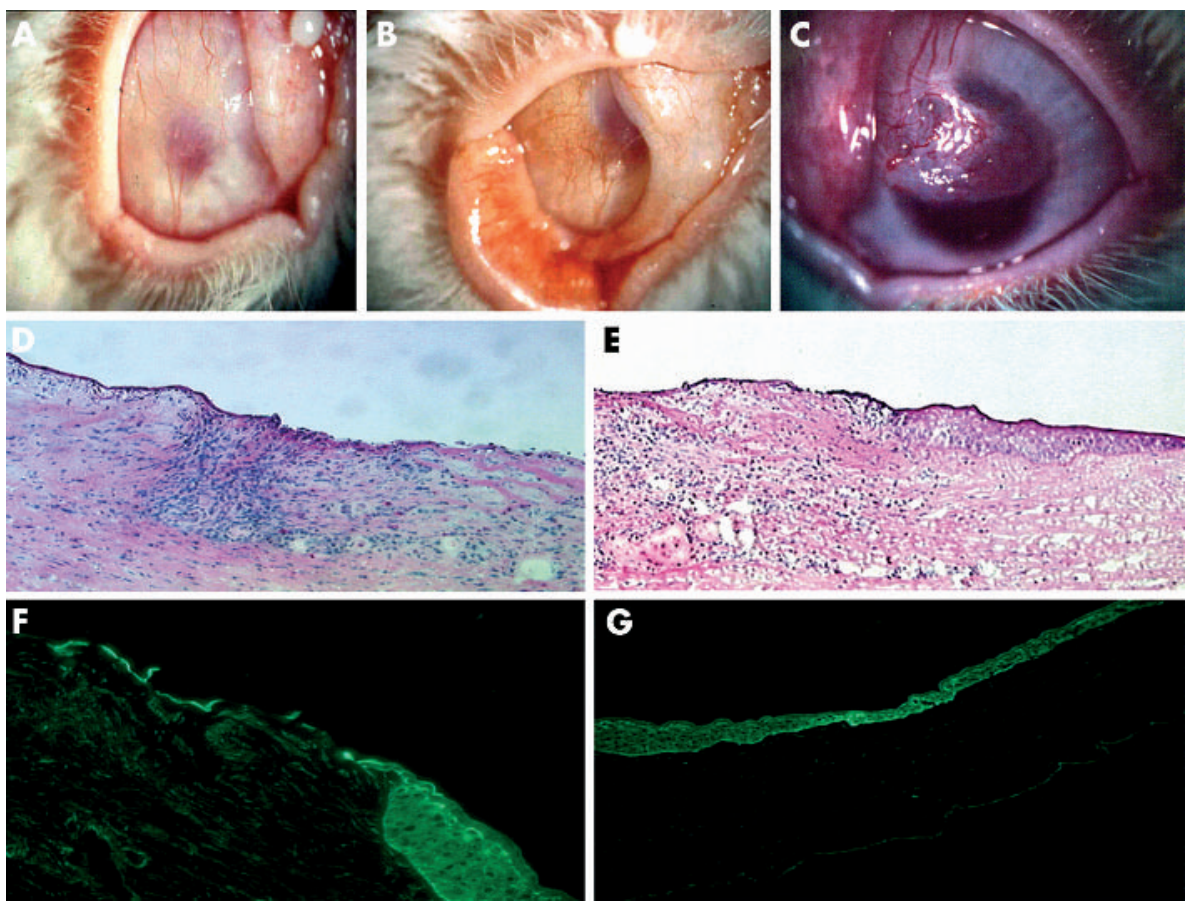

E
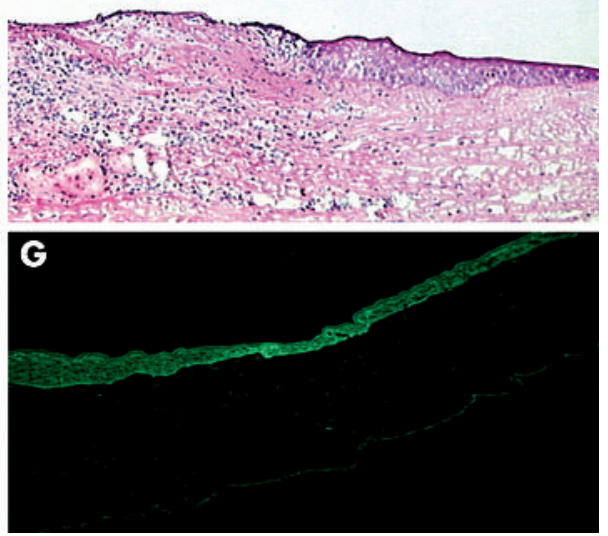

Figure 2 Correlation of clinical partial success with a corneal phenotype and granuloma. In this representative case, the preoperative and postoperative appearance $(\mathrm{A}-\mathrm{C})$. Haematoxylin and eosin staining showed chronic inflammatory cells and blood vessels in pannus ( $D$ and $E$ ). Immunostaining to $\mathrm{K} 3$ keratin was negative in the pannus region $(F, G)$. strated the recovery of a normal stratified corneal epithelium, a PAS positive new basement membrane, and an avascular stroma with uniform cellularity (fig lD, E). In some corneas, the amniotic basement membrane was preserved in the transplanted corneas as a thick eosinophilic layer (fig lD, bracket). Immunostaining to $\mathrm{K} 3$ keratin showed full thickness positivity in the entire corneal epithelium (inset, fig $\mathrm{IF}$ ) and suprabasal positivity in the limbal region (arrow, fig $\mathrm{lF}$ ), while immunostaining to MUC5AC showed conjunctival goblet cells only in the conjunctival epithelium (fig lG). This pattern is identical to the normal corneal epithelial phenotype in vivo and denoted as "corneal (K) phenotype."

In groups II, III, and IV, some corneas with clinical partial success had a smooth and avascular surface and clear stroma in most of the cornea (fig $2 \mathrm{~A}-\mathrm{C}$ ), while part of the cornea was covered with vascularised pannus (fig 2C). Haematoxylin and eosin staining showed that the pannus region was covered by an attenuated epithelium and the stroma was infiltrated with chronic inflammatory cells and blood vessels (fig 2D, E). Immunostaining revealed that the majority of the corneal surface had a K phenotype (fig 2F, G), while the pannus region was devoid of $\mathrm{K} 3$ expression (fig $2 \mathrm{~F}$ ). The pannus was also negative to MUC5AC staining (not shown), and conjunctival goblet cells were found only in the conjunctiva (not shown). Therefore, we still graded these corneas as a corneal (K) phenotype (table 1$)$. In contrast, most corneas with clinical partial success actually showed a mixture of corneal and conjunctival phenotypes on the corneal surface. One such example is shown in figure 3, where a stratified epithelium was found in one part of the cornea (fig 3D, E), while the other part covered by vascularised pannus, contained a thinner epithelium with goblet cells under haematoxylin and eosin staining. This mixed phenotype (denoted as M) was verified by the presence of both full thickness K3 keratin (fig 3F, G) and MUC5AC positive goblet cells (fig $3 \mathrm{H}$ ) on the corneal surface.

For all groups, corneas with clinical failure continued to be covered by vascularisation and an irregular epithelium (fig 4A, B). Among them, a small portion of corneas showed $M$ phenotype as defined in figure 3, but the majority of them showed a conjunctival $(\mathrm{J})$ phenotype. Under haematoxylin and eosin staining, the epithelium was not as organised and

Table 1 Correlation between clinical and phenotypic outcomes

\begin{tabular}{|c|c|c|c|c|c|c|c|c|}
\hline \multirow[b]{2}{*}{ Rabbit } & \multicolumn{2}{|l|}{ Group I } & \multicolumn{2}{|l|}{ Group II } & \multicolumn{2}{|c|}{ Group III } & \multicolumn{2}{|c|}{ Group IV } \\
\hline & Clinical & Phenotype & Clinical & Phenotype & Clinical & Phenotype & Clinical & Phenotype \\
\hline 1 & $\mathrm{~F}$ & $\mathrm{~J}$ & $\mathrm{~F}$ & $M$ & $\mathrm{~F}$ & $\mathrm{~J}$ & PS & $\mathrm{K}$ \\
\hline 2 & $\mathrm{~F}$ & $\mathrm{~J}$ & PS & $\mathrm{J}$ & $\mathrm{F}$ & $M$ & $S$ & K \\
\hline 3 & $\mathrm{~F}$ & J & $\mathrm{F}$ & J & $\mathrm{F}$ & $\mathrm{J}$ & PS & $M$ \\
\hline 4 & $\mathrm{~F}$ & $M$ & $\mathrm{~S}$ & $\mathrm{~K}$ & $\mathrm{~F}$ & $\mathrm{~J}$ & $S$ & $\mathrm{~K}$ \\
\hline 5 & $\mathrm{~F}$ & $\mathrm{~J}$ & $\mathrm{~F}$ & $\mathrm{~J}$ & $\mathrm{~F}$ & $\mathrm{~J}$ & $\mathrm{~S}$ & $\mathrm{~K}$ \\
\hline 6 & $\mathrm{~F}$ & $\mathrm{~J}$ & $\mathrm{~F}$ & $M$ & PS & $M$ & $\mathrm{~S}$ & K \\
\hline 7 & $\mathrm{~F}$ & J & $S$ & $\mathrm{~K}$ & $S$ & $\mathrm{~K}$ & PS & $\mathrm{K}$ \\
\hline 8 & $\mathrm{~F}$ & J & $\mathrm{F}$ & $M$ & $\mathrm{~F}$ & J & $S$ & $\mathrm{~K}$ \\
\hline 9 & $\mathrm{~F}$ & $\mathrm{~J}$ & $\mathrm{~F}$ & $M$ & $\mathrm{~F}$ & $M$ & PS & $M$ \\
\hline 10 & $\mathrm{~F}$ & $\mathrm{~J}$ & $\mathrm{~F}$ & $M$ & $\mathrm{~F}$ & $\mathrm{~J}$ & PS & $\mathrm{K}$ \\
\hline 11 & & & $\mathrm{~F}$ & $\mathrm{~J}$ & $\mathrm{~F}$ & $\mathrm{~J}$ & $S$ & $\mathrm{~K}$ \\
\hline 12 & & & $\mathrm{~F}$ & $M$ & $\mathrm{~F}$ & $\mathrm{~J}$ & $\mathrm{~F}$ & $\mathrm{~J}$ \\
\hline 13 & & & $\mathrm{~F}$ & $\mathrm{~J}$ & $\mathrm{~F}$ & J & & \\
\hline 14 & & & $\mathrm{~F}$ & J & PS & $\mathrm{K}$ & & \\
\hline 15 & & & $S$ & $M$ & $S$ & $\mathrm{~K}$ & & \\
\hline
\end{tabular}



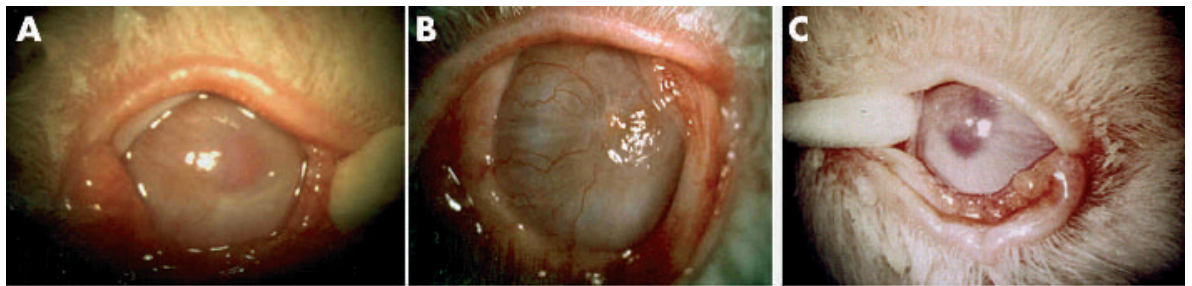

Figure 3 Correlation of clinical partial success with a mixed phenotype. In this representative case, preoperative and postoperative appearance $(A-C)$. Haematoxylin and eosin staining $(D$ and E). Immunostaining to K3 keratin (F and $\mathrm{G})$, and MUC5AC positive goblet cells $(\mathrm{H})$ on the corneal surface.
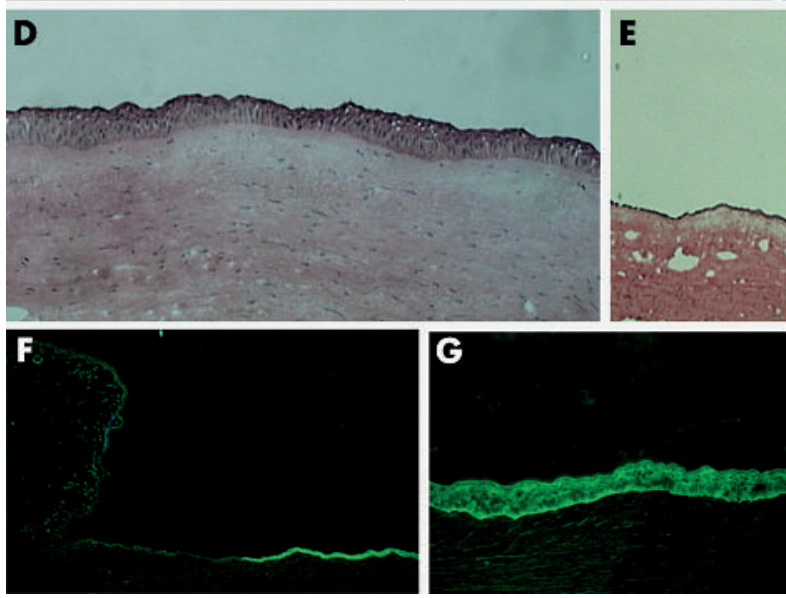

stratified, the basement membrane was indistinct, and the stroma was infiltrated with blood vessels and chronic inflammatory cells (fig 4C). Immunostaining showed negative K3 keratin staining (fig 4D), but positive MUC5AC stained goblet cells throughout (fig 4E).

\section{Correlation of clinical and phenotypic outcomes}

Table 1 summarises clinical and phenotypic outcomes of all four groups. None of group I (AMT alone, $n=10$ ) had clinical success. In groups II, III, and IV $(\mathrm{n}=42)$, there were a total of 11 clinical successes $(p<0.0001)$. Except for one, which had a

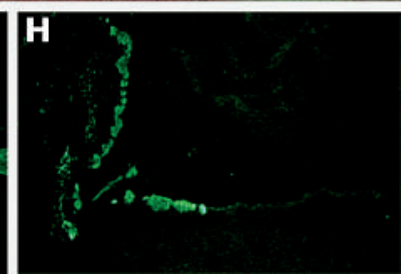

conjunctival phenotype, the rest 10 had a corneal phenotype. None of group I had partial clinical success either. However, in groups II, III, and IV, there were a total of eight clinical partial successes, of which four corneas had a corneal phenotype, three had a mixed phenotype, and one had a conjunctival phenotype. All 10 eyes in group I showed clinical failures. Except for one eye, which had a mixed phenotype, the rest (nine) had a conjunctival phenotype. In groups II, III, and IV, there were a total of 23 clinical failures, of which 15 eyes had a conjunctival phenotype, and eight eyes had a mixed phenotype. Clinical failure correlated with a conjunc-
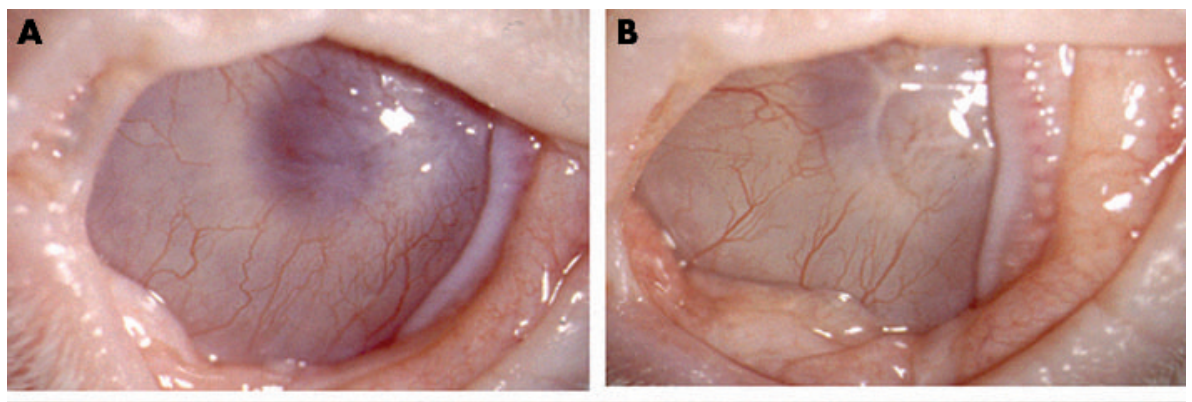

Figure 4 Correlation of clinical failure with a conjunctival phenotype. In this representative case, preoperative and postoperative appearances $(A, B)$ haematoxylin and eosin staining $(C$ arrow marks goblet cells).

Immunostaining showed negative K3 keratin staining (D), but positive MUC5AC stained goblet cells throughout (E).
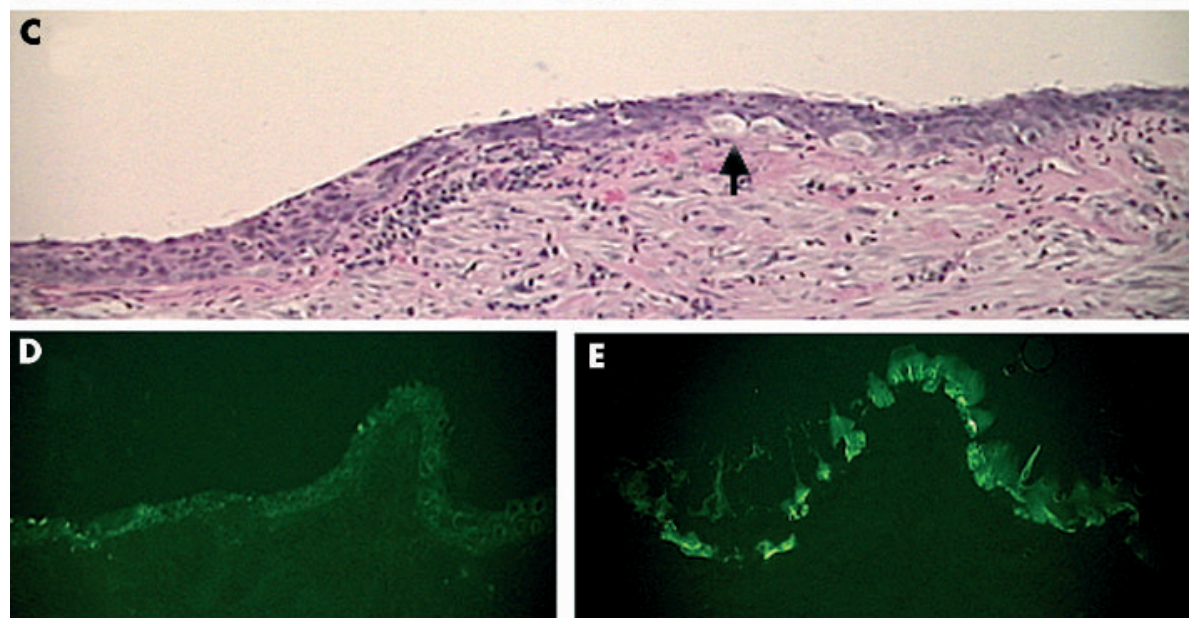
tival phenotype $(\mathrm{p}<0.001)$, while clinical success correlated with a corneal phenotype $(\mathrm{p}<0.01)$. Clinical partial success could be either a corneal phenotype or a mixed phenotype $(p<0.05)$. When the phenotypic outcome was used to assess the efficacy of this procedure, we noted that the conjunctival phenotype was significantly more in group $\mathrm{I}(\mathrm{p}<0.003)$, while the corneal phenotype was significantly more in groups II-IV $(\mathrm{p}<0.05)$, especially in group IV.

\section{DISCUSSION}

Previously we reported that autologous limbal epithelial cells expanded ex vivo on amniotic membrane is effective in restoring a normal corneal surface in this rabbit model of total LSCD. ${ }^{12}$ In this study, we provided additional evidence to support that such clinical successes correlated well with the restoration of a non-keratinised stratified epithelium without goblet cells (fig $1 \mathrm{D}$ and $\mathrm{E}$ ), and a recovery of a corneal epithelial phenotype based on the expression of corneal epithelium specific K3 keratin (fig IF) and the lack of expression of conjunctival goblet cell specific MUC5AC (fig $1 G$ ). As reported, ${ }^{12}$ clinical success was not found in any of 10 eyes $(0 \%)$ of group I with amniotic membrane alone, but was found in 11 of 42 eyes (26\%) in groups II, III, and IV $(\mathrm{p}<0.0001)$ with additional ex vivo expanded limbal epithelial cells. Among these 11 eyes, 10 (91\%) had a corneal epithelial phenotype, confirming that ex vivo expanded limbal epithelial cells were responsible for the restoration of a normal corneal epithelial phenotype. This finding is consistent with our laboratory data showing that amniotic membrane cultures help expand limbal epithelial SC. ${ }^{21-23}$ Because such a clinical success and a corneal phenotype lasted for more than 1 year after transplantation, we further confirmed that ex vivo expansion of limbal epithelium is a valid procedure to treat unilateral total LSCD. Because a significantly higher percentage of clinical success and corneal phenotype was noted in group IV, we also believe protection of newly transplanted limbal epithelium by another amniotic membrane is crucial to ensure this success.

In contrast, clinical failures correlated well with a conjunctival epithelial phenotype, verified by the lack of K3 keratin expression (fig 4D) but positive expression of MUC5AC (fig 4E). As reported, ${ }^{12}$ clinical failures were noted in all 10 eyes of group I, of which all except one had a conjunctival epithelial phenotype. Clinical failures were also noted in 23 of 42 eyes of groups II, III, and IV. We noted that clinical failure correlated with a conjunctival epithelial phenotype $(p=0.001)$. Among these 23 eyes, 15 eyes $(65 \%)$ had a conjunctival phenotype, while the rest (eight) eyes (35\%) had a mixed phenotype. This result indicated that some clinical failures might not be associated with a complete loss of the corneal epithelial phenotype. Although we cannot resolve whether these residual corneal epithelial cells represent a smaller portion of limbal epithelial SC or simply remaining corneal epithelial TAC (transient amplifying cells), we did learn from our earlier report ${ }^{12}$ that these failures correlate well with the early epithelial defects in the first 3 months after transplantation because of exposure problems in groups II and III.

In groups II, III, and IV, four of eight eyes (50\%) showed a corneal epithelial phenotype (fig 2), suggesting that the pannus noted clinically was not caused by "conjunctivalisation" but rather by "granuloma." Therefore, judging from the phenotypic outcome, the success rate was further elevated to $75 \%$. Three of eight eyes (38\%) showed a mixed phenotype (fig 3), suggesting that the pannus noted in these eyes was caused by focal conjunctivalisation-that is, partial LSCD. Differentiation of partial LSCD from granuloma by impression cytology is clinically important to guide effective therapies.
These end point outcome analyses did not reveal the biological process during the wound healing. For example, successful corneas showed progressive wound remodelling from an opaque amniotic membrane to transparent corneal stroma. We thus speculate that transplantation of corneal epithelial cells aids the return of stromal clarity. Further studies of such wound healing processes leading to the restoration of corneal stroma will help us understand how scarless wound healing may be achieved in the cornea.

\section{ACKNOWLEDGEMENTS}

Supported by Public Health Service Research Grant EY06819 (to SCGT via TissueTech, Inc) from Department of Health and Human Services, National Eye Institute, National Institute of Health, Bethesda, Maryland, Human Manpower Development Program, Singapore National Eye Centre, Singapore (to SET), Research Fellowship grant GR-1814/1-2 (to MG) by the Deutsche Forschungsgemeinschaft, Bonn, Germany, and a grant from Ocular Surface Research and Education Foundation.

\section{Authors' affiliations}

S-E Ti, M Grueterich, E M Espana, A Touhami, S C G Tseng, TissueTech, Inc, and Ocular Surface Center, Miami, FL, USA

M Grueterich, D F Anderson, S C G Tseng, Bascom Palmer Eye Institute, University of Miami School of Medicine, Miami, FL, USA

S-E Ti, Singapore National Eye Centre, Singapore

Proprietary interest: SCGT and his family are more than $5 \%$ shareholders of TissueTech, Inc, which owns US patents Nos 6,152,142 and $6,326,019$ on the method of preparation and clinical uses of human amniotic membrane distributed by Bio-Tissue, Inc.

Presented in part at the annual meeting of ARVO in Ft Lauderdale, FL, USA, in May 2003

\section{REFERENCES}

1 Schermer A, Galvin S, Sun T-T. Differentiation-related expression of a major $64 \mathrm{~K}$ corneal keratin in vivo and in culture suggests limbal location of corneal epithelial stem cells. J Cell Biol 1986;103:49-62.

2 Cotsarelis G, Cheng SZ, Dong G, et al. Existence of slow-cycling limbal epithelial basal cells that can be preferentially stimulated to proliferate: implications on epithelial stem cells. Cell 1989;57:201-9.

3 Tseng SCG. Regulation and clinical implications of corneal epithelial stem cells. Mol Biol Rep 1996;23:47-58.

4 Puangsricharern V, Tseng SCG. Cytologic evidence of corneal diseases with limbal stem cell deficiency. Ophthalmology 1995;102:1476-85.

5 Tseng SCG. Conjunctival grafting for corneal diseases. In: Tasman W, Jaeger EA, eds. Duane's clinical ophthalmology. Philadelphia: JB Lippincott, 1994:1-11.

6 Holland EJ, Schwartz GS. The evolution of epithelial transplantation for severe ocular surface disease and a proposed classification system. Cornea 1996;15:549-56.

7 Pellegrini G, Traverso CE, Franzi AT, et al. Long-term restoration of damaged corneal surface with autologous cultivated corneal epithelium. Lancet 1997;349:990-3.

8 Tsai RJF, Li L-M, Chen J-K. Reconstruction of damaged corneas by transplantation of autologous limbal epithelial cells. N Engl J Med 2000;343:86-93.

9 Koizumi N, Inatomi T, Suzuki T, et al. Cultivated corneal epithelial transplantation for ocular surface reconstruction in acute phase of StevensJohnson syndrome. Arch Ophthalmol 2001;119:298-300.

10 Koizumi N, Inatomi T, Suzuki T, et al. Cultivated corneal epithelial stem cell transplantation in ocular surface disorders. Ophthalmology 2001; 108:1569-74.

11 Grueterich M, Espana EM, Touhami A, et al. Phenotypic study of a case with successful transplantation of ex vivo expanded human limbal epithelium for unilateral total limbal stem cell deficiency. Ophthalmology 2002; 109:1547-52.

12 Ti S-E, Anderson DF, Touhami A, et al. Factors affecting outcome following transplantation of ex vivo expanded limbal epithelium on amniotic membrane for total limbal deficiency in rabbits. Invest Ophthalmol Vis Sci 2002;43:2584-92.

13 Kruse FE, Chen JJY, Tsai RJF, et al. Conjunctival transdifferentiation is due to the incomplete removal of limbal basal epithelium. Invest Ophthalmol Vis Sci 1990;31:1903-13.

14 Huang AJW, Tseng SCG. Corneal epithelial wound healing in the absence of limbal epithelium. Invest Ophthalmol Vis Sci 1991;32:96-105.

15 Tseng SCG. Staging of conjunctival squamous metaplasia by impression cytology. Ophthalmology 1985;92:728-33. 
16 Lee S-H Tseng SCG Amniotic membrane transplantation for persistent epithelial defects with ulceration. Am J Ophthalmol 1997; 123:303-12.

17 Meller D, Tseng SCG. Conjunctival epithelial cell differentiation on amniotic membrane. Invest Ophthalmol Vis Sci 1999:40:878-86.

18 Tsai RJF, Sun T-T, Tseng SCG. Comparison of limbal and conjunctival autograft transplantation for corneal surface reconstruction in rabbits. Ophthalmology 1990;97:446-55.

19 Kim JC, Tseng SCG. Transplantation of preserved human amniotic membrane for surface reconstruction in severely damaged rabbit corneas. Cornea $1995 ; 14: 473-84$
20 Chen JJY, Tseng SCG. Corneal epithelial wound healing in partial limbal deficiency. Invest Ophthalmol Vis Sci 1990;31:1301-14.

21 Meller D, Pires RTF, Tseng SCG. Ex vivo preservation and expansion of human limbal epithelial stem cells on amniotic membrane cultures. Br J Ophthalmol 2002;86:463-71.

22 Grueterich M, Tseng SCG. Human limbal progenitor cells expanded on intact amniotic membrane. Arch Ophthalmol 2003 (in press).

23 Grueterich M, Espana E, Tseng SC. Connexin 43 expression and proliferation of human limbal epithelium on intact and denuded amniotic membrane. Invest Ophthalmol Vis Sci 2002;43:63-71. 